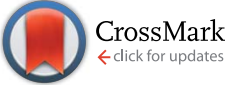

Cite this: Chem. Sci., 2015, 6, 6525

\title{
Structural basis of head to head polyketide fusion by CorB $\dagger$
}

\author{
Georg Zocher, $\dot{t}^{\mathrm{a}}$ Joachim Vilstrup, $\dot{\dagger}^{\mathrm{b}}$ Daniel Heine, ${ }^{\mathrm{c}}$ Asis Hallab, ${ }^{\mathrm{d}}$ Emilie Goralski, ${ }^{\mathrm{e}}$ \\ Christian Hertweck, ${ }^{\text {cf }}$ Mark Stahl, ${ }^{9}$ Till F. Schäberle ${ }^{\star e}$ and Thilo Stehle*ah
}

Corallopyronin A is a polyketide derived from the myxobacterium Corallococcus coralloides with potent antibiotic features. The gene cluster responsible for the biosynthesis of corallopyronin $A$ has been described recently, and it was proposed that CorB acts as a ketosynthase to interconnect two polyketide chains in a rare head-to-head condensation reaction. We determined the structure of CorB, the interconnecting polyketide synthase, to high resolution and found that CorB displays a thiolase fold. Sitedirected mutagenesis showed that the catalytic triad consisting of a cysteine, a histidine and an asparagine is crucial for catalysis, and that this triad shares similarities with the triad found in HMG-CoA synthases. We synthesized a substrate mimic to derivatize purified CorB and confirmed substrate attachment by ESI-MS. Structural analysis of the complex yielded an electron density-based model for the polyketide chain and showed that the unusually wide, T-shaped active site is able to accommodate two polyketides simultaneously. Our structural analysis provides a platform for understanding the unusual head-to-head polyketide-interconnecting reaction catalyzed by CorB.

Received 9th July 2015

Accepted 6th August 2015

DOI: $10.1039 /$ c5sc02488a

www.rsc.org/chemicalscience antibiotics, antiparasitic, immunosuppressive, and antitumoral agents. ${ }^{2,3}$

Corallopyronin A (Cor) is a polyketide isolated from the myxobacterium Corallococcus coralloides. ${ }^{4,5}$ Cor contains an $\alpha$ pyrone entity and shows promising antibiotical activity in vivo by targeting the so-called hinge region of the bacterial DNAdependent RNA polymerase (RNAP), thereby preventing the initiation of transcription. ${ }^{6}$ Cor is thought to interact with conserved RNAP residues, thus exhibiting broad-spectrum antibacterial activity while leaving the eukaryotic transcription machinery intact. In addition, Cor interacts with RNAP residues that are remote from the binding sites of other characterized RNAP inhibitors, e.g. rifamycin, thus remaining active towards rifamycin-resistant bacteria. ${ }^{6,7}$ Cor is produced by Corallococcus coralloides strains, which are gliding, heterotrophic myxobacteria originally isolated from soil samples. ${ }^{8}$ As Cor shows substantial activity against various pathogenic bacteria including methicillin-resistant staphylococci (MRSA), it represents a promising lead compound for antibacterial drug development. ${ }^{9}$ While the activity of Cor against mycobacteria is only moderate, this is regarded as an advantage for the treatment of diseases such as lymphatic filariasis and onchocerciasis without concern for cross-resistance development in tuberculosis. ${ }^{9}$ These diseases affect over 150 million people in over 80 developing countries of the tropics and subtropics. They are caused by filarial nematodes, and it was recognized that depleting the obligate endosymbionts, i.e. Wolbachia, from the worms results in cure of the patients. Recently, Cor was shown to possess superior in vivo antimicrobial activity against

\$ These authors contributed equally to the work. 
Wolbachia. ${ }^{5}$ Thus, the compound is currently in the focus of a translational project that aims to establish Cor as a new antibiotic drug. ${ }^{9}$

The gene cluster responsible for the biosynthesis of Cor was described recently, ${ }^{4}$ and the authors showed by stable-isotope labeling experiments that two polyketide chains are synthesized, interconnected, and subsequently modified resulting in the formation of Cor (Fig. 1). ${ }^{4}$ Based on sequence analysis, CorB was assigned as a $\beta$-ketoacyl-ACP-synthase I. It was proposed that CorB performs an unusual head-to-head condensation reaction to interconnect two polyketide chains. Polyketide synthases (PKS) catalyzing head-to-head connections are rare but were described for plant type III PKSs. ${ }^{\mathbf{1 0 1 1}}$ Ketosynthases usually catalyze the Claisen condensation of acylthioesters with malonylthioesters in a head-to-tail reaction, yielding an elongated carbon chain. Different classes of ketosynthases have been described according to the architecture and function of the system: (i) in modular type I PKSs, ketosynthases are part of large multifunctional enzymes in which a nascent chain is passed on from module to module, whereas (ii) in type II PKSs, the units are loosely associated, iteratively working, stand-alone enzymes, and (iii) in type III PKSs the enzymes are multifunctional and act iteratively. ${ }^{12}$ In addition to polyketide systems, ketosynthases are also crucial in fatty acid biosynthesis. ${ }^{13}$ In contrast to the headto-tail fusion catalyzed by ketosynthases, hydroxymethylglutarylCoA synthases (HMG-CoA synthases) catalyze the condensation of acetoacetyl-CoA with a $\beta$-keto thioester such as acetoacetylCoA. In this way, 3-hydroxy-3-methylglutaryl-CoA (HMG-CoA) is formed in mevalonate-dependent isoprenoid pathways. ${ }^{\mathbf{1 4}}$ Although the reaction is comparable to the biosynthesis of polyketides, the enzymatic reaction of HMG-CoA synthases lacks the decarboxylation process that is a common feature of the condensing enzymes in the thiolase superfamily. ${ }^{15}$ Irrespective of the different types and variants of ketosynthases, all of them share the thiolase fold. ${ }^{16}$ The reaction mechanism varies among enzymes of this superfamily. However, with a few exceptions, ${ }^{17,18}$ most superfamily members catalyze the formation of a carboncarbon bond between a polyketide chain bound as a thioester to

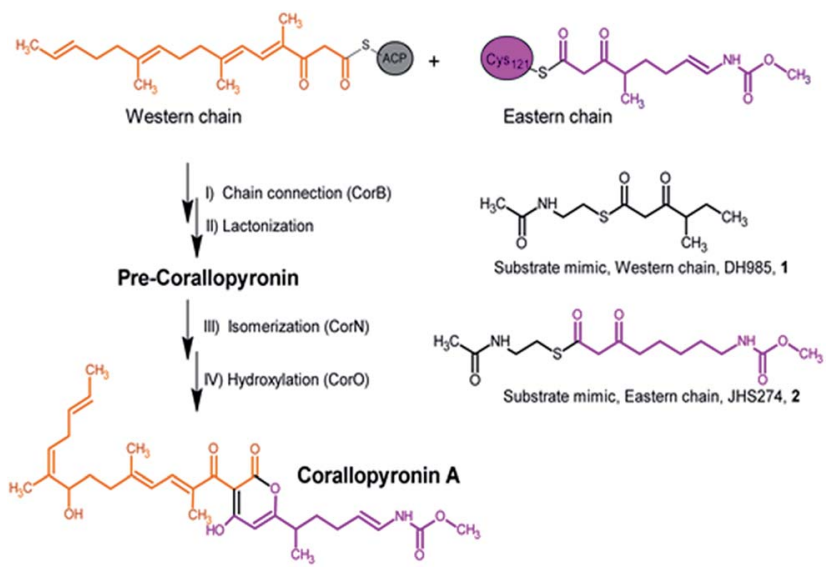

Fig. 1 Section of the biosynthesis of corallopyronin A and the catalyzed reaction of CorB including the substrate mimics used in the in vitro enzyme assay. an activated cysteine and an $\alpha$-carboxylated extender unit bound as a thioester to either an acyl carrier protein (ACP) or coenzyme $\mathrm{A}(\mathrm{CoA}){ }^{15}$

Here we report a structural and biochemical analysis of CorB, a member of a novel class of head-to-head condensing ketosynthases involved in the biosynthesis of $\alpha$-pyrone antibiotics such as Cor. The enzyme is encoded by a stand-alone gene within the biosynthetic gene cluster. With an in vitro assay, we show that CorB catalyzes the interconnection of the two precursor chains (Fig. 1) with a Claisen-like condensation reaction. The subsequent lactonization eventually yields the central pyrone ring system of Cor.

\section{Results and discussion}

\section{Substrate analogue synthesis and CorB activity assay}

CorB was heterologously produced in E. coli. Due to the lack of commercially available substrate analogues, we synthesized mimics of the natural substrates. According to our hypothesis for pyrone ring formation ${ }^{4}$ the substrates should carry a $\beta$-keto residue. Moreover, natural substrates are tethered to a phosphopantetheine (PPant) arm, which represents the essential prosthetic group of carrier proteins within PKS/NRPS systems. We therefore synthesized substrate analogue $\mathbf{1}$ as a $\mathrm{N}$-acetylcysteamine (SNAC) thioester derivative (Fig. 1, S2 and S3 $\dagger$ ). Substrate 1 was designed to mimic both the eastern and the western chain, as these differ exclusively in the saturation when regarding the carbon skeleton up to atom C5. We assumed that this substrate mimic choice would be a reasonable compromise to investigate CorB binding. Together with substrate mimic $2{ }^{19}$ these have been the substrates for the in vitro assay to test the enzymatic Claisen condensation reaction. In this way, the PPant arm is mimicked, and the molecules are activated for the subsequent reactions.

Purified CorB was assayed using 1 and 2 as substrates. In the presence of freshly purified CorB, the $m / z$ of the expected product methyl (5-(4-hydroxy-3-(2-methylbutanoyl)-2-oxo-2 $\mathrm{H}$ pyran-6-yl)pentyl) carbamate 3 was detected using high resolution LC-MS. (Fig. 2). To form the pyrone ring, the first of the two SNAC-substrates is covalently bound to C121 of CorB (compare with Fig. 1), whereas the other chain - in vivo ACP-tethered - is still bound to SNAC. Then, CorB catalyzes the interconnection of both chains and releases the SNAC entity (of ACP in vivo). This is followed by lactonization, yielding a closed pyrone ring and liberating the product. In control reactions, either without a substrate, or without CorB, 3 was not detected.

\section{Phylogenetic analysis}

A set of 415 highly homologous proteins was selected from the NCBI database of prokaryotic genomes. On the basis of these data, a strict consensus tree was reconstructed using maximum likelihood and parsimony algorithms (Fig. 3). ${ }^{20,21}$ A clear separation of the ketosynthases into distinct clades was observed. The clade comprising CorB includes many proteins annotated as $\beta$-ketoacyl-(acyl-carrier-protein) synthase III (FabH) proteins, and forms a sister clade to proteins related to $\beta$-ketoacyl-(acyl- 
(a)

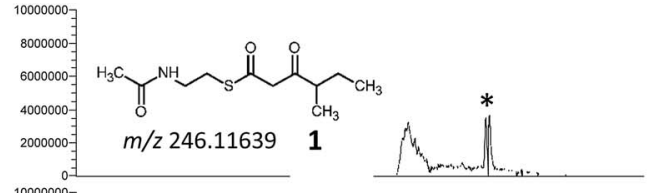

2

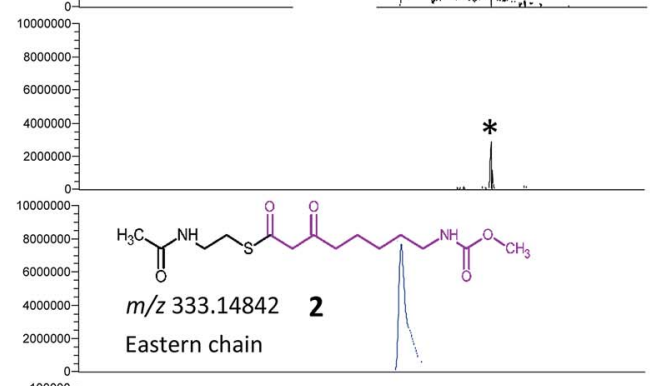

4

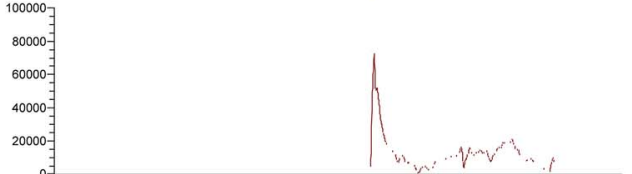

5

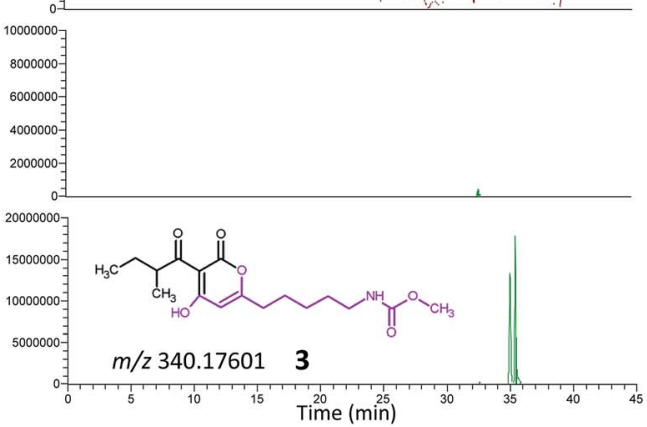

(b)
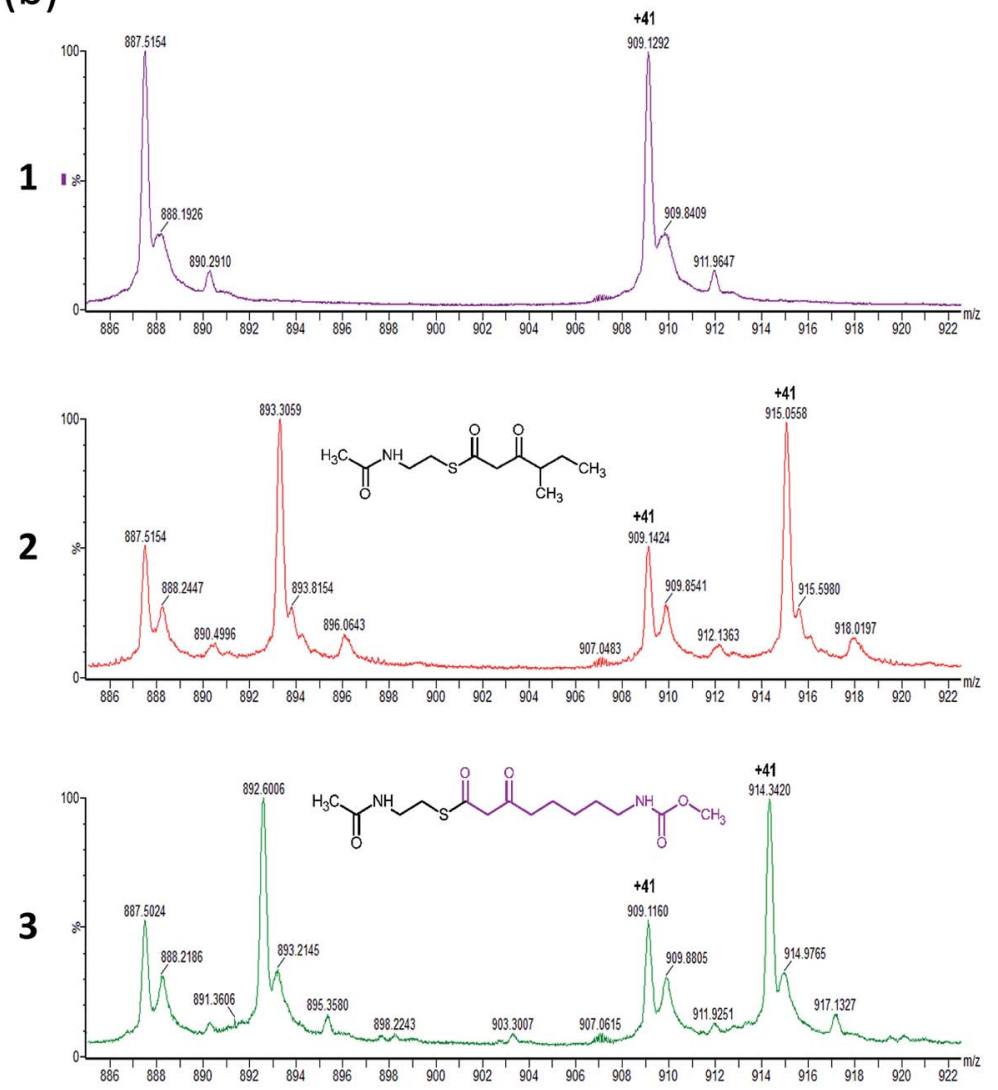

Fig. 2 Results of the CorB in vitro activity assay (a) and ESI-MS experiment of CorB used in the crystallization experiment. (a) Extracted ion chromatograms for the assay (traces 2,4 , and 6 ) and the respective control reaction without CorB (traces 1,3 , and 5 ). The extracted $m / z$ of substrate 1 (trace 1 and 2) and substrate 2 (trace 3 and 4), as well as for product 3 (trace 5 and 6 ) are given. In presence of the CorB protein the concentration of the educts decreased and the product was formed. The asterisk marks a contamination that is similar in mass but not related to substrate 1. The impurity is not present in trace 5 and 6 due to screening for a different mass. (b) Section of the ESI-MS experiments. All three samples resulted from an identical setup including the same protein and differ exclusively in the presence or absence of the substrates. The results for the wildtype CorB (panel 1), CorB incubated with 1 (panel 2), and CorB incubated with 2 (panel 3) are depicted.

carrier-protein) synthase I (FabB). Likewise, the recently biochemically analyzed ketosynthases $\mathrm{CerJ}^{7}$ and DarB $^{22}$ belong to distinct clades. In the context of CorB it is notable that MyxB, the corresponding KS encoded in the myxopyronin biosynthetic gene cluster of Myxococcus fulvus, ${ }^{23}$ is not the closest homologue. This finding is surprising because of the high structural similarity of the $\alpha$-pyrone antibiotics produced by the myxobacteria C. coralloides and M. fulvus. The core region of the corallopyronins and the myxopyronins is identical; solely the western chain (Fig. 1) exhibits some differences. In Cor, carbon C-24 is hydroxylated and the chain is elongated, comprising seven more carbon atoms than myxopyronin A. Even though CorB and MyхB are responsible for the chain interconnection prior to pyrone ring formation (this study and Sucipto et al., respectively), the phylogeny indicates that some minor differences exist between these enzymes. However, both enzymes harbor the Cys-His-Asn catalytic triad characteristic for FabH and type III PKSs instead of the Cys-His-His triad found in ketosynthases of type I PKSs. Twelve out of 15 ketosynthase (KS) and KS-like protein(domain)s encoded within the corallopyronin gene locus (Fig. S4†) possess this Cys-His-Asn triad.

\section{The structure of CorB}

As CorB catalyzes the polyketide-interconnecting reaction, we sought to determine the structure of CorB to analyze the architecture of the active site and to gain insight into the determinants of specificity for the formation of the pyrone ring. We produced pure CorB and crystallized it for X-ray structure determination. We solved the structure of the native, dimeric protein to $1.6 \AA$ resolution (Table $\mathrm{S} 1 \dagger$ ) by molecular replacement and confirmed that CorB assumes a typical thiolase fold (Fig. S5 $†$ ). ${ }^{16}$ Additionally, we observed that the catalytic cysteine C121 was partially oxidized (CorB-ox) (Fig. S6a $\dagger$ ). This oxidation would hamper the incorporation of our substrate mimic as it dramatically changes the charge distribution of the reactive sulfur atom, and therefore the nucleophilicity of the active cysteine. As attempts to reduce the catalytic cysteine were unsuccessful, we optimized protein production and crystallization to prevent oxidation. The resulting structure (CorB-red) did not show significant oxidation, as evidenced by the lack of additional electron density near $\mathrm{C} 121$ (Fig. S6B $\dagger$ ), and we next derivatized CorB through addition of $\mathbf{1}$. The resulting structure 


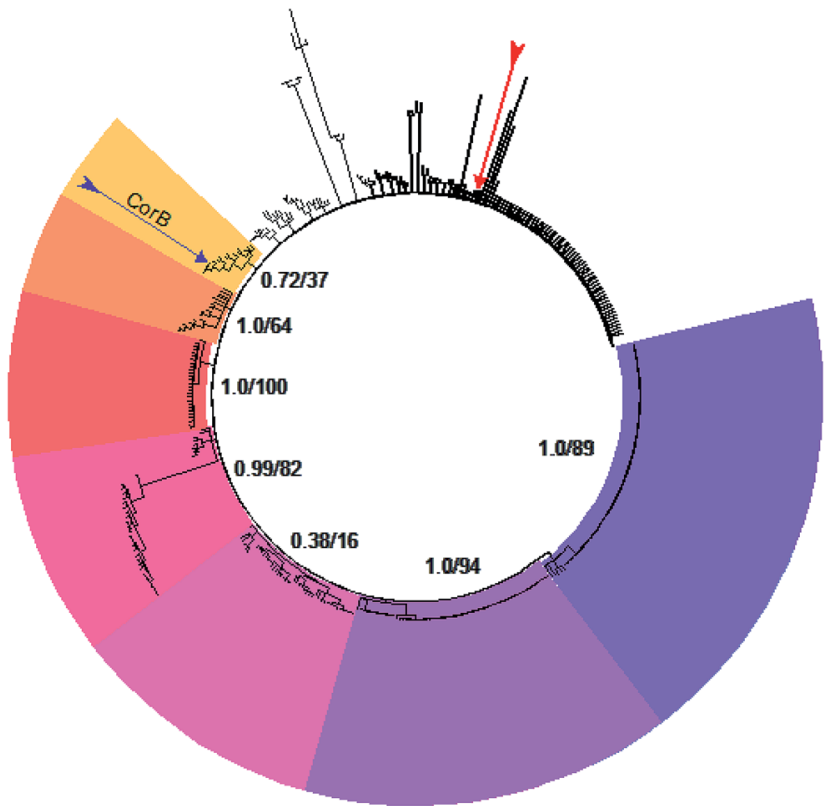

Fig. 3 Consensus tree of selected ketosynthases. The different clades are coloured. CorB (marked by the purple arrow) is situated in the yellow clade. These proteins show highest homology to FabH. Starting in the yellow clade, the homology to FabB (dark purple clade) increases counterclockwise. The red arrow marks MyxB, the enzyme catalyzing the CorB-corresponding chain interconnection in myxopyronin biosynthesis. The support values of the respective clade (maximum likelihood and parsimony, respectively) are given.

(CorB-1) clearly showed positive electron density around the catalytic cysteine as a result of substrate binding (Fig. S6C $\dagger$ ). Since the electron density was not clearly contoured, we confirmed the successful attachment of the substrate mimic by an ESI-mass experiment. The CorB sequence translates into a theoretical mass of 37 233.6 Da. The ESI mass spectrum of the wildtype protein (Fig. 2) results in an experimental mass of $37233.2 \mathrm{Da}$, thus confirming the integrity and identity of the CorB protein. After incubation of CorB with 1, we observed a mass increase of 244 Da by ESI-mass spectroscopy. This mass increase corresponds well with the total mass of the entire substrate 1 (theoretical mass $245 \mathrm{Da}$ ) (Fig. 1 and S7 $\dagger$ ), and it therefore shows that $\mathbf{1}$ attaches to CorB.

\section{Structure comparison to other thiolase enzymes}

As a member of the thiolase superfamily CorB features a typical thiolase fold characterized by two $\beta \alpha \beta \alpha(\beta) \alpha \beta \beta$ motifs. As described for CerJ, ${ }^{17}$ CorB lacks one $\beta$-strand ( $\beta 8$ for FabH from E. coli) in the C-term $\beta \alpha \beta \alpha(\beta) \alpha \beta \beta$ motif (Fig. 4 and S5 $\dagger$ ). Another feature of the dimeric CorB structure is a deep, widely accessible T-shaped channel that results mainly from a fairly open conformation of helix $\alpha 4$, loop $\beta 6 \alpha 4$, the residues connecting $\beta 3$ with $\beta 4$ (residues 152-166) and $\beta 1$ with $\beta 2$ (residues $27-46$ ). As expected, a structural similarity search ${ }^{24}$ identified several $\mathrm{FabH}$ enzymes from different organisms as close structural homo$\log \mathrm{s}^{25-28}$ with $\mathrm{C} \alpha$ atom rms deviations of around $2.5 \AA$ (Table S2 $\dagger$ ). The core fold of the thiolase is very well conserved among these proteins, and main differences occur in the part of the protein that is responsible for the T-shaped tunnel of the active site of CorB (see above). In addition to FabH enzymes, a structural comparison revealed several PKS enzymes ${ }^{29-33}$ (Table $\mathrm{S} 3 \dagger$ ) and 3-hydroxy-3-methylglutaryl-coenzyme A (HMG-CoA) synthases as structurally homologous enzymes. Although the core motif is very well conserved and the quality of the CorB alignment is comparable between the two groups, the more open shape of CorB fits best to the HMG-CoA synthases. We conclude this from two observations. First, residues between $\beta 3 \beta 4$ and $\beta 1 \beta 2$ show an open conformation as found in HMGCoA synthases, whereas the PKS and FabH enzymes exhibit a tighter active site as a result of a closed setup of the corresponding residues (Fig. S8 $\dagger$ ). Second, helix $\alpha 5$, which is tilted in CorB compared with all structurally related proteins, is also shifted towards the active site by approximately $2 \AA$, placing the tip of the helix (P266) at a similar position to that found in the HMG-CoA enzymes.

\section{Comparison of the catalytic triad}

The active site of CorB contains a catalytic triad formed by C121, $\mathrm{H} 264$ and N294. These three residues are arranged in a clamp geometry that is reported to stabilize the carbon chain elongation process during fatty acid biosynthesis or polyketide formation. ${ }^{34-36}$ All FabH and PKS enzymes possess this catalytic triad. CerJ has to be excluded from the active site comparison as it features a sequential rather than a clamp-like catalytic triad observed in cysteine proteases. ${ }^{17}$ The structural similarity of the CorB active site especially to those of HMG-CoA synthases is remarkable and agrees well with the biochemical data available for PKS and FabH enzymes on the one hand and CorB and HMG-CoA enzymes on the other. The catalysis of FabH and PKS enzymes is accompanied by a decarboxylation step to form the nucleophilic carbon atom that is subsequently attached to the electrophilic carbonyl atom linked as a thioester to the catalytic cysteine residue. This decarboxylation step is not observed in the family of HMG-CoA. ${ }^{37-39}$ For HMG-CoA synthases, this is mainly because the third residue of the triad (Asn) is not involved in substrate stabilization. A closer look reveals the structural reason for the difference in the chemical reaction. In HMG-CoA synthases, helix $\alpha 5$ is shifted towards the active center by about $1.2 \AA$, and this helix carries a proline (P266) at its C-terminus. A search in the non-redundant UniProt database showed that this proline is strictly conserved in all HMG-CoA synthases but not found in FabH and PKS enzymes. The steric repulsion of the proline residue is the major reason for the separation of both clamp residues (His-Asn) of the catalytic triad, and thus a small but significant distance increase (Fig. 2b). A more detailed investigation comparing the distances of the triad residues (Fig. 5) in the FabH, PKS and HMG-CoA enzymes showed that the shortest mean distances are observed for the PKS and FabH enzymes. In the group of the HMG-CoA synthases, the mean distance of the asparagine to the triad members is increased by at least $1.1 \AA$ A. Such a change in the distance would substantially weaken a postulated hydrogen bond and therefore makes it plausible that the decarboxylation 

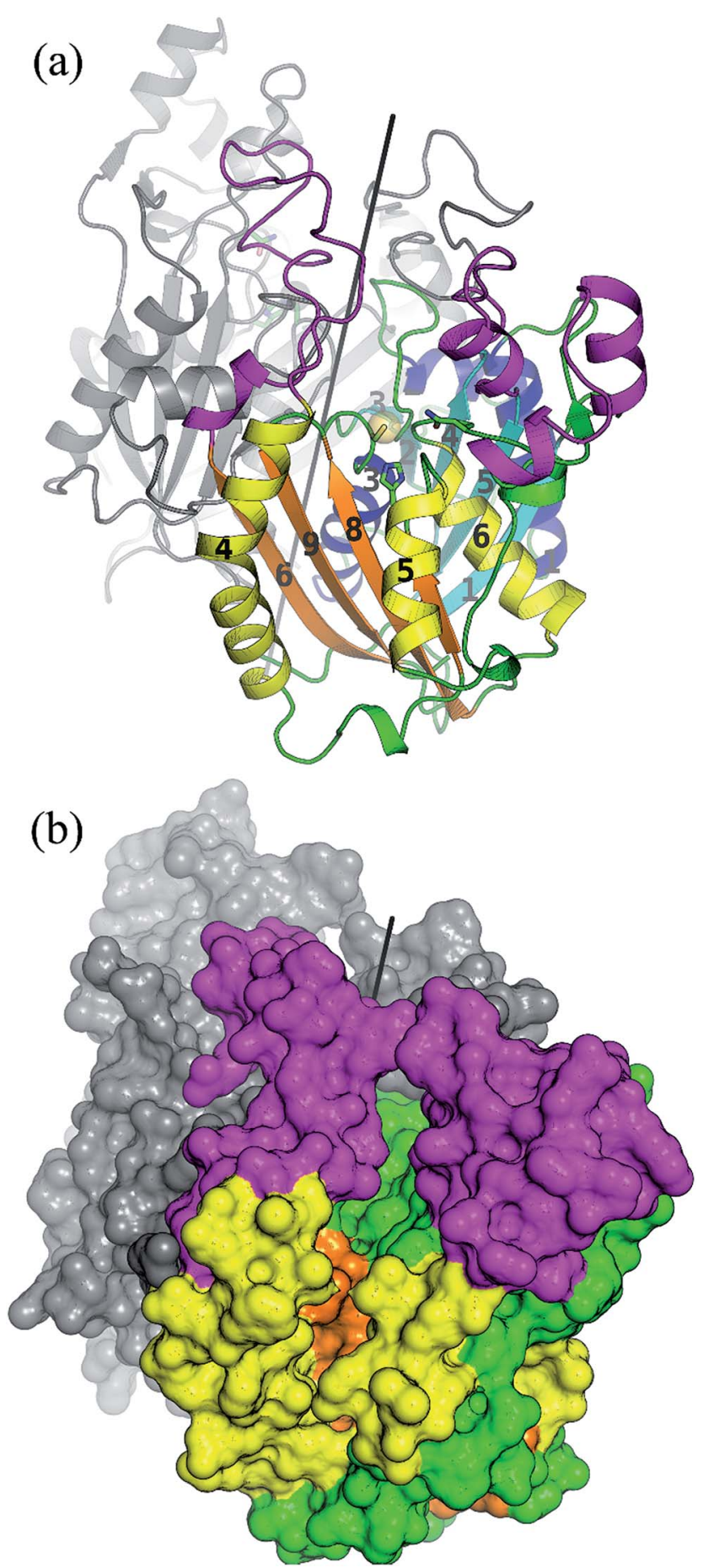

Fig. 4 Overall structure of the dimeric CorB enzyme reveals a classical thiolase fold. Both characteristic $\beta \alpha \beta \alpha(\beta) \alpha \beta \beta$ motifs of this superfamily are coloured by their secondary structural elements in cyan and blue for the $\mathrm{N}$-terminal motif and orange and yellow for the $\mathrm{C}$-terminal motif. The regions exhibiting larger deviations to structural homologue enzymes are coloured in pink. The catalytic triad is represented as ball and sticks and the reactive sulfur atom of C121 is emphasized by a yellow sphere. The dimeric two-fold axis (parallel to the crystallographic two-fold axis) is shown in black. (b) Surface representation revealing a wide $\mathrm{T}$-shaped active site cavity of CorB. process is disfavoured by the loss of stabilization. Indeed, a hydrogen bond to the corresponding asparagine residue (N326) is not reported in the complex structure of the HMG-CoA synthase from Brassica juncea with acetyl-CoA. ${ }^{38}$ With a $\mathrm{H} 264-(\mathrm{C} \gamma$ atom)-to-N292-(C $\gamma$ atom) distance of $8.8 \AA$, the residues of the catalytic triad of CorB-1 are even farther apart (Fig. 5). Although decarboxylation is chemically not expected due to the substrate specificity of CorB, the opened active site of CorB clearly correlates with the increased separation of the catalytic residues.

To investigate the relevance of the members of the catalytic triad for catalysis, we performed site-directed mutagenesis of CorB, and investigated several single residue mutants (ESI $\dagger$ ). These point mutations were introduced by two-step PCR with primers carrying the desired mutation, and the resulting constructs were verified by DNA sequencing. In particular, the three amino acids forming the catalytic triad $\mathrm{C}-\mathrm{H}-\mathrm{N}$ were mutated to C121A, C121S, H264A, H264F, and N292A. In all mutants, product formation was not observed using the established assay, in contrast to the wt enzyme. These results demonstrate that all three triad residues participate in the reaction catalyzed by CorB, and they in particular show that N292 is required for catalysis, in contrast to what has been reported for HMG-CoA synthase. ${ }^{37-39}$

\section{Binding of the substrate mimic to CorB}

The active site of CorB forms a T-shaped cavity. The anterior opening forms the entrance to the active site, which is loaded with CoA-linked acyl-entities. This is in good agreement with the available structural data of thiolase enzymes in complex with acetylated CoA. ${ }^{40,41}$ The catalytic cysteine C121 is found in the center of a deep cleft at the bottom of the cavity. Next to the catalytic cysteine, H264 and N292 are found on the lateral site. Opposed to them, the oxyanion hole is formed by the amide backbone of S324. Elongated channels can be found on both sides of the catalytic center. The proximal cavity is flanked by primarily hydrophobic residues (Fig. 6) and runs towards the two-fold dimeric interface. Residues 93 to 95 and 206 to 208 of the adjacent protomer contribute to the formation of this channel. In chalcone synthases and type II polyketide synthases, this channel harbors the so-called gatekeeper residues that are thought to control the chain length of the nascent polyketide chain. ${ }^{42-44}$ In HMG-CoA synthases, this channel is blocked by a set of rigid residues. ${ }^{38,39,45}$ The proximal cavity of CorB is also much larger compared to another reported 2pyrone synthase. ${ }^{35}$ This is in good agreement with the reported biochemistry of this 2-pyrone synthase, which generates a rather small product, 6-methyl-4-hydroxy-2-pyrone, through the iterative condensation of malonyl entities. In addition to 2pyrone synthases, the proximal cavity is also reported to accommodate substrates in PKS11 of Mycobacterium tubercu${ }^{l o s i s^{31}}$ and the anthraniloyl-skeleton in PqsD. ${ }^{46}$ In contrast to PKS11 and PqsD, the cavity in CorB is substantially enlarged towards the dimeric interface. This differs compared to the anthranilate binding site in PsqD where the cavity is sealed in that direction by an intensive $\pi-\pi$-stacking of residues F205, 


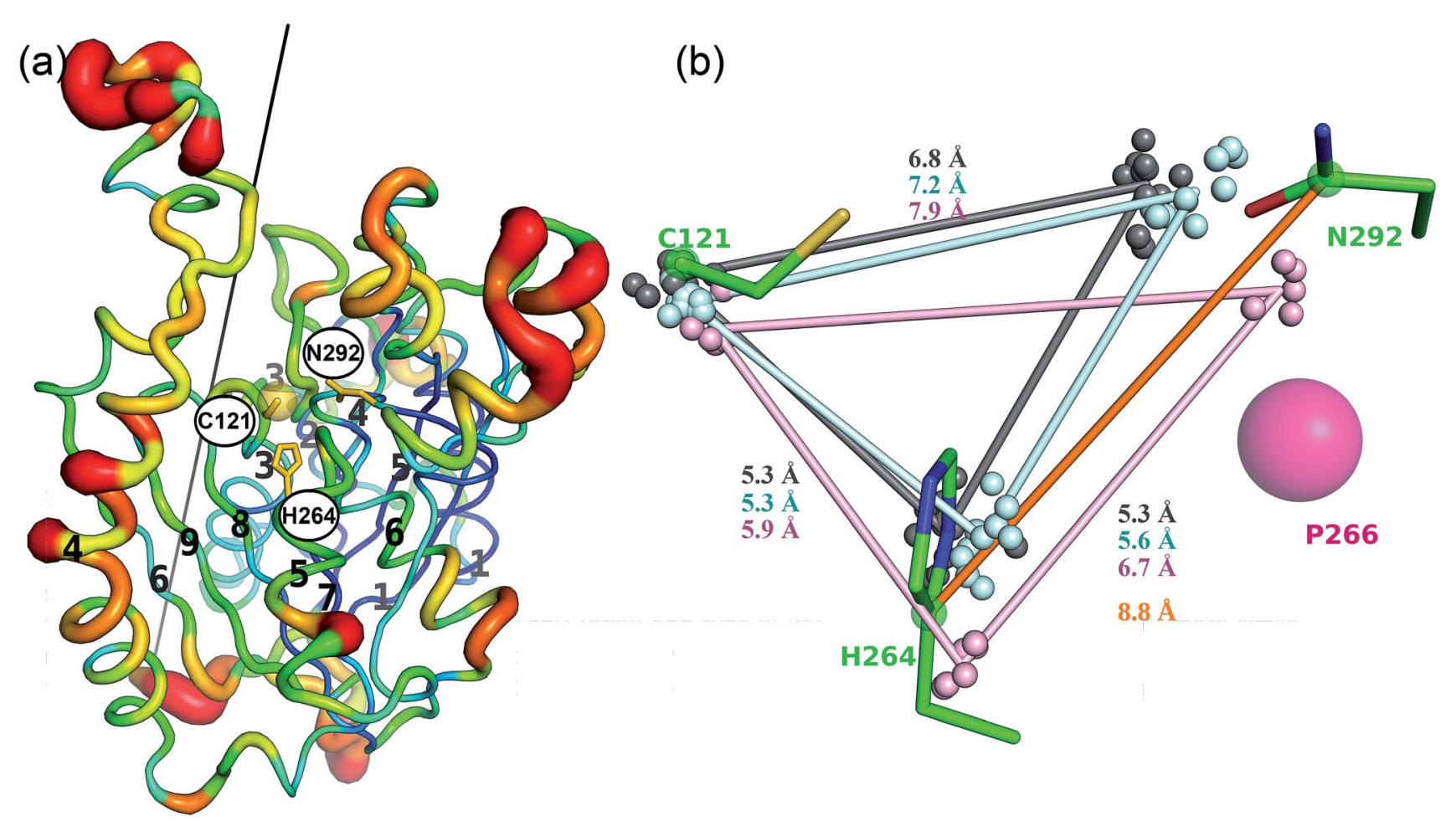

Fig. 5 Structural comparison of CorB with related enzymes. (a) Superposition of CorB with 15 non-redundant homologous enzymes. The protein chain is colored according to the mean $C \alpha$-rmsd deviation for this superposition, ranging from $0.3 \AA$ (blue) to $4 \AA$ (red). As expected larger deviations occur at the surface. The $\mathrm{N}$-terminal $\beta \alpha \beta \alpha(\beta) \alpha \beta \beta$ motif is structurally more conserved compared to the $\mathrm{C}$-terminal part, in good agreement with the dimeric structure of CorB that is formed by residues in the N-terminal region. The black line shows the two-fold axis that generates the biological CorB dimer. (b) The distances separating the catalytic triad residues of CorB are substantially larger compared with the decarboxylating enzymes FabH (grey) and type-III PKS enzymes (cyan), and they are more similar to the HMG-CoA synthases (violet). The C $\alpha$ atoms of the catalytic cysteines and the $\mathrm{C}_{\gamma}$-atoms of the catalytic histidines or asparagines are shown as small spheres for the structural homologs. With a H264-( $\mathrm{C}_{\gamma}$ atom)-to-N292-( $\mathrm{C}_{\gamma}$ atom) distance of $8.8 \AA$ (orange line), CorB features the largest His-Asn distance.

R145 and F218. This site of the cavity is blocked by the bulky side chain of tryptophan W230 in PKS11. Interestingly, the architecture of the CorB cavity is closely related to that of FabH from Mycobacterium tuberculosis, ${ }^{\mathbf{4 1}}$ and the binding pocket found for lauroyl-coenzyme A of this enzyme almost perfectly superposes with the cavity of CorB, suggesting that this pocket acts as a substrate channel for the interconnection reaction.

The electron density of derivatized CorB-1§ differs at the active site from that observed for CorB-ox and CorB-red. For CorB-ox we found that the catalytic cysteine is oxidized to cysteine-S-dioxide (Fig. S6A $\dagger$ ). This is likely a result of crystal growth, which initially took several days and facilitated oxidation of the reactive sulfur atom of C121. Optimization of the purification and crystallization protocol then yielded crystals overnight. These crystals allowed us to obtain a structure that clearly shows a reduced cysteine at the active site (CorB-red). For CorB-1, which was also crystallized with the optimized protocol, we observed a substantial positive electron density at C121 as a result of substrate incubation prior to crystallization. We also attempted crystallization of CorB incubated with substrate 2 but unfortunately could not produce suitable crystals for structure determination. As the crystallization conditions are similar and both structures, CorB-1 and CorB-red, result from the same protein batch, we conclude that the positive density at C121 is due to substrate binding. Although all structures are virtually identical ( $\mathrm{C} \alpha$-rms deviation of $0.4 \AA$ ), the oxidation of the cysteine in CorB-ox led to drastic change in its electrochemical properties and hampered the covalent linkage to the substrate. Thus, we conclude that CorB-ox is inactive. Although the electron density for the substrate observed in CorB-1 is weak and ambiguous (Fig. S6C $\dagger$ ), we confirmed the attachment of the substrates 1 and 2 by ESI-mass spectroscopy. The experiments were performed using the same protein batch and identical solutions, incubation times and temperatures but differ exclusively in the absence or presence of substrate $\mathbf{1}$ or $\mathbf{2}$. We observe a mass difference of 244 Da for 1 (theoretical expected mass increase: $245 \mathrm{Da}$ ) which corresponds well with the intact substrate (Fig. 2B and S9 $\dagger$ ). For 2, we observed a mass increase of $214 \mathrm{Da}$, which corresponds well with the theoretical expected mass increase of $214 \mathrm{Da}$ of the covalently linked substrate lacking the SNAC entity (Fig. $2 \mathrm{~B}$ and S9†). In addition to the electron density around the catalytic cysteine, no extra density features were found either in CorB-ox or CorB-red. We therefore conclude that the mass increase of CorB-1 results from the attachment of substrate 1 to $\mathrm{C121}$. The exact nature of the bound substrate could not be unequivocally determined either by ESI-mass spectroscopy or by X-ray analysis. As the electron density is weak, it does not allow for proper ligand building and 

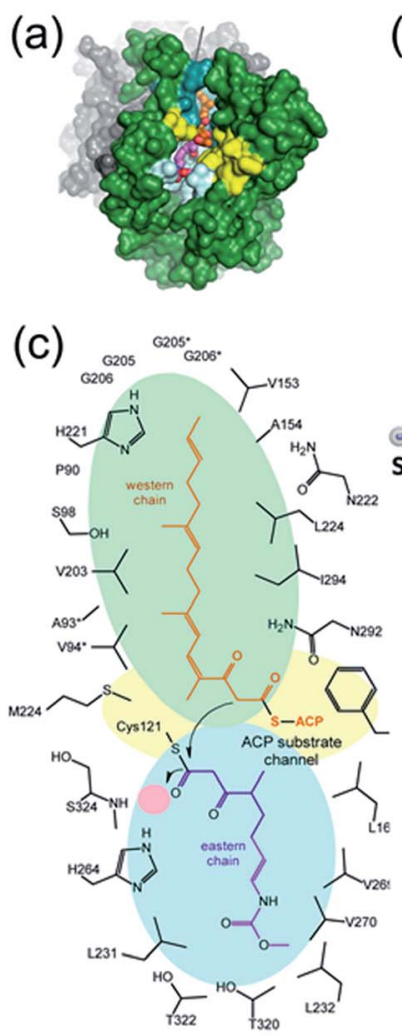

(b)

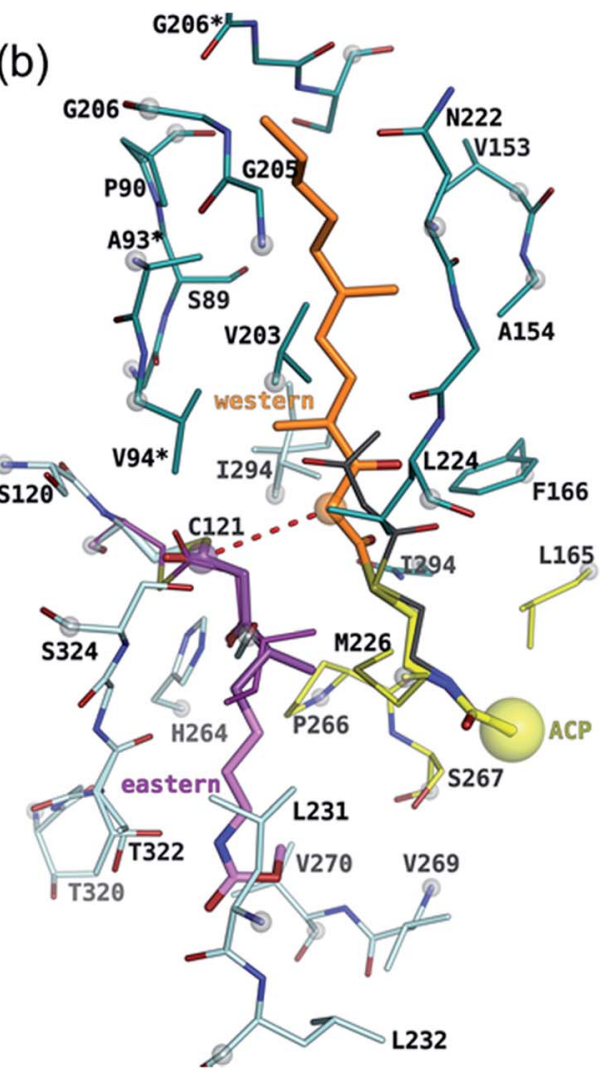

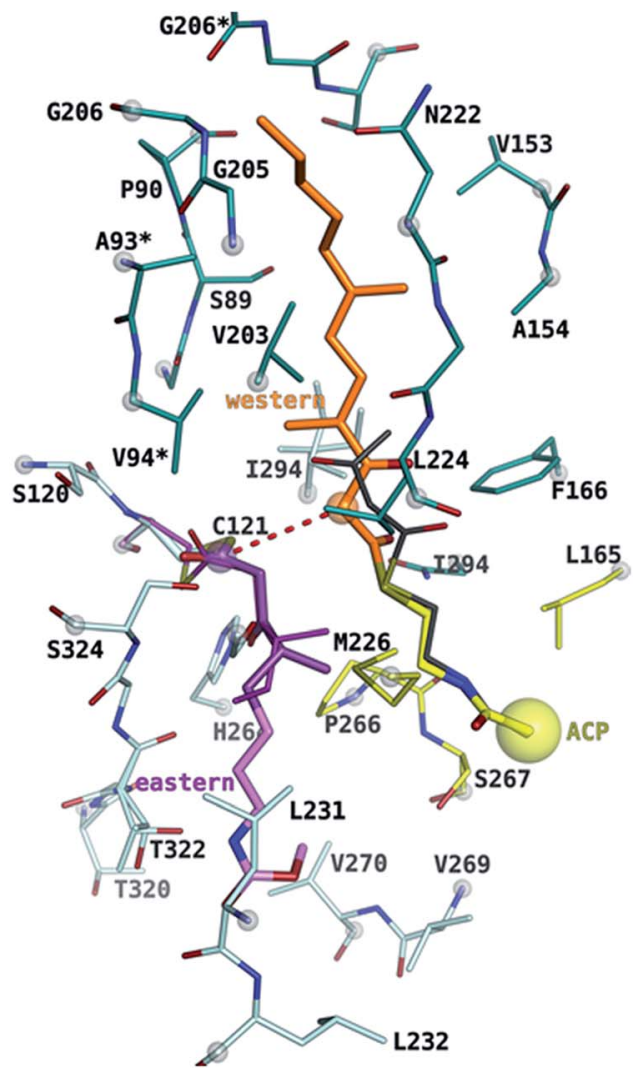

Fig. 6 Proposed model of catalysis of CorB. (a) Overview of the binding site showing the substrates in a ball-and stick representation in the cavity of CorB. (b) Stereo representation of the binding situation. The substrate mimic 1 (dark violet) was placed into the active site on the basis of its unbiased $\left(F_{\circ}-F_{\mathrm{c}}\right)$-difference electron density and the remaining portion of the eastern chain (light magenta) was modeled into the cavity. The western chain was modeled into the proximal cavity on the basis of a homologue 2-pyrone synthase using the pantotheine entity of CoA as an anchor point for the western chain. (c) Schematic view of (b). The oxyanion hole is highlighted by a pink circle.

refinement of the substrate (Fig. S6D †). Nevertheless, the electron density is sufficiently clear to use it as a basis for modelling of substrate 1 into the CorB cavity. Several factors likely contribute to the observed weak electron density. It is possible that the active site is not fully occupied, and the substrate could also have high temperature factors. Moreover the additional state observed by ESI-MS would picture the electron density as an overlay of both states yielding a lower quality.

With respect to the difference in electron density, we placed 1 into the cavity of CorB in two slightly different conformations (Fig. S6C $\dagger$ ). The distal channel is formed by a set of mostly hydrophobic residues (L231, L232, V269, V270 and T320). The substrate model would suggest a hydrogen bond to H264. This is in good agreement with our mutation studies, which show that a replacement of $\mathrm{H} 264$ with either alanine or phenylalanine would result in a loss of activity. The binding direction of $\mathbf{1}$ is comparable to the situation found in a eukaryotic HMG-CoA synthase covalently attached to an irreversible inhibitor, ${ }^{38}$ thus supporting the mode of binding of the eastern chain.

\section{Proposed catalytic mechanism}

As a ternary complex of CorB with both substrates (eastern and western chain) could not be generated, we modelled the second polyketide (western chain) into the active site of CorB-1. For this, we compared several enzymes of the thiolase family in complex with acyl-CoA molecules. ${ }^{35,40,41}$ We superposed the CoA recognition site of another 2-pyrone synthase ${ }^{35}$ (2-PS) and FabH from Mycobacterium tuberculosis ${ }^{41}$ with the corresponding residues in CorB (residues 44-47 and 161-166) and found that the acyl moiety would point into the proximal cavity (Fig. 6). On the basis of this structural comparison, we placed a model of the western chain into the proximal cavity of CorB using the CoA PPant entity of the structure relative as the anchor point for the western chain. The western chain would fit into the cavity without steric clashes.

This model of substrate binding is in good agreement with the observed biochemistry. In our model, the eastern chain is covalently linked to the catalytic cysteine in the first step of catalysis and occupies the distal channel of CorB. The covalent attachment to the catalytic cysteine activates the eastern chain. In the second step, the western chain is placed into the proximal cavity, orienting the $\alpha$-carbon in a position suitable for the nucleophilic attack by the activated eastern chain. The nucleophilicity of the western chain might be enhanced by an interaction with $\mathrm{N} 292$, which was determined to be crucial for catalysis. The resulting intermediate is stabilized by the oxyanion hole formed by the backbone amide of S234, and the polyketide connection reaction proceeds with the release of the 
catalytic cysteine. We suggest that the lactonization process is a spontaneous reaction that takes place in solution after dissipation as the lacton formation is accompanied with sterical requirements that are unlikely to take place in the active site of CorB.

Our insights into Cor biosynthesis will facilitate future derivatization approaches of $\alpha$-pyrone antibiotics using combinatorial biosynthesis. Usually, modifications of an original natural product are advantageous in terms of pharmacokinetics. However, Cor and its derivatives represent difficult-tosynthesize compounds since chemical synthesis is not feasible on a commercial scale. ${ }^{47}$ Now, in silico and in vitro experiments become possible to determine the substrate flexibility of CorB. This forms a promising template for the rational design of optimized producer strains for the further development of the compound, e.g. as anthelmintic.

\section{Experimental}

\section{Cultivation of strains}

B035 was cultivated in casitone medium (MD1 medium, supplemented with $0.2 \%$ glucose). MD1 medium consists of casitone $3 \mathrm{~g} \mathrm{~L}^{-1}, \mathrm{CaCl}_{2} \times 2 \mathrm{H}_{2} \mathrm{O} 0.7 \mathrm{~g} \mathrm{~L}^{-1}, \mathrm{MgSO}_{4} \times 7 \mathrm{H}_{2} \mathrm{O} 2 \mathrm{~g} \mathrm{~L}^{-1}$. The flasks were agitated on a rotary shaker $(140 \mathrm{rpm})$ at $30^{\circ} \mathrm{C}$ for 7-14 days. $E$. coli strains were cultivated in LB medium (tryptone $10 \mathrm{~g}$, yeast extract $5 \mathrm{~g}, \mathrm{NaCl} 10 \mathrm{~g}$, filled with $\mathrm{H}_{2} \mathrm{O}$ to $1 \mathrm{~L}$; for solid medium $1.6 \%$ agar was added) supplemented with $100 \mu \mathrm{g} \mathrm{mL}$ ampicillin (Amp) for selection and to maintain the constructs.

\section{Molecular-biological methods}

Molecular-biological work was performed according to standard procedures. Polymerase chain reactions (PCRs) were performed with oligonucleotides obtained from eurofins genomics and are listed in Table S4. $\dagger$ DNA isolation from agarose gels was performed using either the Wizard ${ }^{\circledR}$ SV Gel and PCR Clean-Up System (Promega), or the QIAquick Gel Extraction Kit (Qiagen). Plasmid isolation was performed using either the PureYield ${ }^{\mathrm{TM}}$ Plasmid Miniprep System kit (Promega), or the QIAprep Spin Miniprep Kit (Qiagen).

\section{DNA manipulation}

Either genomic DNA or plasmid DNA served as template for the amplification of DNA fragments. Genomic DNA isolation of $C$. coralloides B035 is described in Erol et al., 2010. ${ }^{4}$ In order to obtain the expression construct for CorB a fragment of $1012 \mathrm{bp}$ was amplified using the primer pair CorB_TOPO_up and CorB_TOPO_dn. This fragment was introduced into the vector pET151TOPO by directional TOPO ${ }^{\circ}$ cloning of the blunt-ended PCR products. Then, the resulting vector was transferred in $E$. coli One Shot®TOP10 cells by transformation. The resulting plasmid was isolated and checked for identity by sequencing. Plasmid with the desired sequence was transferred into competent cells of the expression host E. coli BL21 (DE3) Star by transformation. Subsequently, cells were plated on LB agar supplemented with ampicillin for selection.

\section{Site-directed mutagenesis}

To introduce point mutations into the enzyme, a two-step PCR was applied. Two primers were designed carrying the point mutation. In a first round the point mutation was introduced into the two amplified fragments using (i) the forward primer CorB_TOPO_up and the reverse primer carrying the sequence coding for the amino acid exchange, and (ii) the forward primer carrying the sequence coding for the amino acid exchange and the reverse primer CorB_TOPO_dn (Table $\mathrm{S} 4 \dagger$ ). The two fragments obtained by the first round of PCRs served as templates for the second PCR round. In this second PCR, first 10 cycles were performed without primers in the sample, then the primers CorB_TOPO_up and CorB_TOPO_dn were added, and 20 further cycles were performed. The fragments obtained by the second round of PCR were introduced into pET151TOPO vector by directional TOPO ${ }^{\circledR}$ cloning. Then, the resulting vector was transferred in $E$. coli One Shot ${ }^{\circledR T O P 10}$ cells by transformation. The resulting plasmid was isolated and checked for the presence of the insert by either PCR, or restriction. The identity of positive plasmids was verified by sequencing. Plasmid with the desired sequence was transferred to competent cells of the expression host E. coli BL21 (DE3) Star by transformation. Subsequently, cells were plated on LB agar supplemented with ampicillin for selection.

\section{Protein production}

For the expression of the proteins, the corresponding E. coli strains carrying the desired construct were inoculated in $9 \mathrm{~mL}$ $\mathrm{LB}_{\mathrm{Amp}}$ liquid medium and incubated overnight at $37^{\circ} \mathrm{C}$. With this preculture, the main culture was inoculated in LB medium $(1 \mathrm{~L})$ at $37^{\circ} \mathrm{C}$ until an $\mathrm{OD}_{600}$ of $0.4-0.7$ was reached. Then the cells were cooled down to $16{ }^{\circ} \mathrm{C}$ and protein expression was induced by adding IPTG (final concentration 0.05-0.1 mM). Growth was then continued at $16{ }^{\circ} \mathrm{C}$ overnight. Cells were harvested by centrifugation, resuspended in lysis buffer $(50 \mathrm{mM}$ $\mathrm{NaH}_{2} \mathrm{PO}_{4}, 300 \mathrm{mM} \mathrm{NaCl}, 10 \mathrm{mM}$ imidazole, $\mathrm{pH} 8.0$ ), and transferred to $50 \mathrm{~mL}$ Falcon tubes. The cells were then disrupted by sonification ( 6 cycles à 10 pulses, and cooling on ice during the cycles). After sonification cell debris was removed by centrifugation for $30-45 \mathrm{~min}$ at $8500 \mathrm{rpm}$ at $4{ }^{\circ} \mathrm{C}$. The proteincontaining supernatant was transferred onto gravity flow columns (Qiagen, Germany), which were packed with $1 \mathrm{~mL} \mathrm{Ni-}$ NTA agarose. The flow-through was collected and again loaded onto the columns 3 more times. Then 2 washing steps followed (washing buffer 1: $50 \mathrm{mM} \mathrm{NaH} \mathrm{PO}_{4}, 300 \mathrm{mM} \mathrm{NaCl}, 20 \mathrm{mM}$ imidazole, pH 8.0; washing buffer 2: $50 \mathrm{mM} \mathrm{NaH}_{2} \mathrm{PO}_{4}, 300 \mathrm{mM}$ $\mathrm{NaCl}, 20 \mathrm{mM}$ imidazole, $\mathrm{pH} 8.0$ ). Bound protein was finally eluted with $5 \times 500 \mu \mathrm{L}$ elution buffer $\left(50 \mathrm{mM} \mathrm{NaH}_{2} \mathrm{PO}_{4}, 300 \mathrm{mM}\right.$ $\mathrm{NaCl}, \mathrm{pH}$ 8.0, stepwise increasing imidazole concentration $1 \times$ $100 \mathrm{mM}, 1 \times 150 \mathrm{mM}, 1 \times 200 \mathrm{mM}, 2 \times 300 \mathrm{mM})$. Fractions containing the targeted protein were combined and loaded onto a spin filter column (Millipore, $10 \mathrm{kDa}$ exclusion size) for desalting. This column was centrifuged at $5000 \mathrm{rpm}$ at $4{ }^{\circ} \mathrm{C}$ until only one tenth of the starting volume remained. Then the protein was re-buffered in either Tris buffer (50 mM Tris, $\mathrm{pH}$ 
8.0), or Tris buffer containing DTT and glycerol (20 mM Tris, 1 mM DTT, and 5\% glycerol, $\mathrm{pH}$ 7.5).

The protein production for crystallization experiments was essentially similar but instead $E$. coli Rosetta 2 cells were used for protein expression at $20^{\circ} \mathrm{C}$. The cell pellet was resuspended in His-A buffer ( $20 \mathrm{mM}$ TrisHCl (pH 8.8), $0.5 \mathrm{M} \mathrm{NaCl}, 10 \mathrm{mM}$ imidazole and $2 \mathrm{mM}$ DTT). After further centrifugation (18 000 $\left.\mathrm{rpm}, 1 \mathrm{~h}, 4^{\circ} \mathrm{C}\right)$ the supernatant was loaded onto a $\mathrm{Ni}^{2+}$-affinity column ( $5 \mathrm{~mL}$ HisTrapFF GE Healthcare $\left.{ }^{\mathrm{TM}}\right)$ in a cyclic manner. The column was connected to an Äkta purifier (GE Healthcare $^{\mathrm{TM}}$ ) and proteins were eluted by applying a step gradient of His-B buffer (20 mM TrisHCl (pH 8.8), $0.5 \mathrm{M} \mathrm{NaCl}, 500 \mathrm{mM}$ imidazole and $2 \mathrm{mM}$ DTT). Fractions containing CorB were pooled and dialyzed against SEC buffer $(20 \mathrm{mM}$ TrisHCl $(\mathrm{pH}$ 8.8), $150 \mathrm{mM} \mathrm{NaCl}$ ) for $18 \mathrm{~h}$ at $4{ }^{\circ} \mathrm{C}$. After buffer exchange, TEV protease was added at a $1 / 10$ mass ratio $(\mathrm{m} / \mathrm{m})$. The reaction was placed on a merry-go-round for 3 days at $4{ }^{\circ} \mathrm{C}$. Protein digestion was analyzed using SDS-PAGE and the sample was applied onto a Superdex200 16/60 (GE Healthcare ${ }^{\mathrm{TM}}$ ). Fractions containing untagged CorB were pooled, concentrated to $3 \mathrm{mg} \mathrm{mL} \mathrm{m}^{-1}$ and used for crystallographic experiments or flash-frozen in liquid nitrogen for storage at $-80{ }^{\circ} \mathrm{C}$. To obtain reduced CorB all reaction buffers contained reducing agent (TCEP, $5 \mathrm{mM}$ ) beside His-A and His-B buffer.

\section{Synthesis of the substrate analogues}

All reagents were obtained from commercial suppliers (SigmaAldrich and Acros Organics) and were used without further purification. Reactions were performed under an inert atmosphere (Argon) by using the Schlenk technique. All solvents were obtained in HPLC grade and were dried prior to use by the following procedures: dichloromethane (DCM) was distilled from a calcium hydride suspension. Methanol and chloroform were distilled prior to use. For thin-layer chromatography TLC aluminum sheets silica gel $60 \mathrm{~F}_{254}$ (MERCK) were used. Open column chromatography was performed on silica gel 60; particle size 0.015-0.04 mm (Macherey-Nagel).

\section{Synthesis of $S$-(2-acetamidoethyl)4-methyl-3- oxohexanethioate}

A solution of 2-methylbutanoic acid $(200 \mathrm{mg}, 2 \mathrm{mmol})$ in dry dichloromethane $(10 \mathrm{~mL})$ was cooled to $-20{ }^{\circ} \mathrm{C}$ under argon. Meldrum's acid (290 mg, $2 \mathrm{mmol})$, DMAP $(240 \mathrm{mg}, 2 \mathrm{mmol}$ ) and 1-ethyl-3-(3-dimethylaminopropyl)carbodiimide hydrochloride (400 mg, $2 \mathrm{mmol}$ ) were added slowly and the temperature was kept at $-20{ }^{\circ} \mathrm{C}$ for one hour. After the solution was slowly allowed to warm to room temperature, stirring was continued for an additional two hours. Water $(20 \mathrm{~mL})$ was added and the aqueous phase was extracted with dichloromethane $(2 \times 20$ $\mathrm{mL}$ ). The combined organic fractions were dried with $\mathrm{Na}_{2} \mathrm{SO}_{4}$ and concentrated under reduced pressure to give a yellow oil. The crude product and $N$-acetylcysteamine $(240 \mathrm{mg}, 2 \mathrm{mmol})$ were dissolved in toluene $(20 \mathrm{~mL})$ and heated under reflux for two hours. The solvent was removed under reduced pressure and the residue was purified by column chromatography over silica gel (chloroform) to give $350 \mathrm{mg}$ of a clear oil. The title compound exist as a keto : enol mixture $(3: 2)$ in $\mathrm{CDCl}_{3}$. Yield: $71 \% .{ }^{1} \mathrm{H}$ NMR $\left(300 \mathrm{MHz}, \mathrm{CDCl}_{3}\right): \delta 0.86\left(3 \mathrm{H}, \mathrm{t},{ }^{3} J=7.4 \mathrm{~Hz}\right), 0.87$ $\left(3 \mathrm{H}, \mathrm{t},{ }^{3} J=7.4 \mathrm{~Hz}\right), 1.07\left(3 \mathrm{H}, \mathrm{d},{ }^{3} J=6.8 \mathrm{~Hz}\right), 1.10\left(3 \mathrm{H}, \mathrm{d}^{3} J=6.8\right.$ $\mathrm{Hz}), 1.33-1.75(4 \mathrm{H}, \mathrm{m}), 1.94(3 \mathrm{H}, \mathrm{s}), 1.94(3 \mathrm{H}, \mathrm{s}), 2.08(1 \mathrm{H}, \mathrm{ddq}$, ${ }^{3} J_{1}=6.9 \mathrm{~Hz},{ }^{3} J_{2}=6.8 \mathrm{~Hz},{ }^{3} J_{3}=6.8 \mathrm{~Hz}$, enol-form $), 2.53(1 \mathrm{H}, \mathrm{ddq}$, ${ }^{3} J_{1}=6.9 \mathrm{~Hz},{ }^{3} J_{2}=6.8 \mathrm{~Hz},{ }^{3} J_{3}=6.8 \mathrm{~Hz}$, keto-form $), 3.02-3.09(4 \mathrm{H}$, $\mathrm{m}), 3.38-3.48(4 \mathrm{H}, \mathrm{m}), 3.70(2 \mathrm{H}, \mathrm{s}$, keto $), 5.42(1 \mathrm{H}, \mathrm{s}$, enol $), 6.11$ $(2 \mathrm{H}, \mathrm{bs}), 12.60(1 \mathrm{H}, \mathrm{s}$, enol-OH $){ }^{13} \mathrm{C}$ NMR $\left(75 \mathrm{MHz}, \mathrm{CDCl}_{3}\right): \delta$ 11.4, 11.7, 15.3, 17.5, 23.1, 23.1, 25.5, 27.1, 27.8, 29.1, 39.2, 39.9, 41.0, 48.4, 55.4, 98.2, 170.4, 170.6, 181.2, 192.4, 194.4, 206.0 ppm, IR (film): 997, 1085, 1190, 1286, 1544, 1610, 1655, 1718, 2876, 2968, $3288 \mathrm{~cm}^{-1}$, HRMS: (ESI + ): $\mathrm{m} / z$ calculated for $\mathrm{C}_{11} \mathrm{H}_{20} \mathrm{O}_{3} \mathrm{NS}: 246.1158$, found $246.1156[\mathrm{M}+\mathrm{H}]^{+}$.

\section{CorB in vitro assay}

The purified protein was used for the activity assay. A typical assay (final volume $100 \mu \mathrm{L}$ ) consisted of $1 \mu \mathrm{L}$ of each substrate (concentration of 2: $30 \mathrm{mM}$; concentration of 1: $50 \mathrm{mM}$ ) and $0.28 \mathrm{mg}$ protein. The assay was filled up to the final volume by adding the reaction buffer. The latter consisted of $20 \mathrm{mM}$ Tris, 1 $\mathrm{mM}$ DTT, and $5 \%$ glycerol ( $\mathrm{pH}$ 7.5). Negative controls were performed in the same way by omitting protein. Incubation was performed at room temperature. The assay was stopped by adding $200 \mu \mathrm{L}$ of ethyl acetate. The samples were agitated vigorously, and the following phase separation was reached by 2 min centrifugation at full speed in a table top centrifuge. The organic phase was transferred in a new vial, and subsequently dried under nitrogen flow.

\section{UPLC-MS analysis}

Samples were dissolved in $20 \mu \mathrm{L} 50 \%$ acetonitrile (ACN), $0.1 \%$ trifluoroacetic acid (TFA), and $49.9 \% \mathrm{H}_{2} \mathrm{O} .1 .5 \mu \mathrm{L}$ solution were injected onto a C18 trap column $(20 \mathrm{~mm}$ length, $100 \mu \mathrm{m}$ inner diameter, ReproSil-Pur 120 C18-AQ, $5 \mu \mathrm{m}$, Dr Maisch GmbH, Ammerbuch-Entringen, Germany) made in-house. Solvent A was $0.1 \%$ formic acid (FA), solvent B $90 \%$ ACN, $0.1 \%$ FA. The analytical column was equilibrated with $5 \%$ solvent $\mathrm{B}$. The trap column was equilibrated with $1 \%$ ACN, $0.1 \%$ TFA, $98.9 \% \mathrm{H}_{2} \mathrm{O}$. Bound peptides on the trap column were eluted onto a C18 analytical column (150 $\mathrm{mm}$ length, $75 \mu \mathrm{m}$ inner diameter, ReproSil-Pur 120 C18-AQ, $1.9 \mu \mathrm{m})$. Peptides were separated during a linear gradient of $15 \%$ to $80 \%$ solvent $\mathrm{B}$ within $19 \mathrm{~min}$ at $350 \mathrm{~nL} \mathrm{~min}{ }^{-1}$. The nanoHPLC was coupled online to an LTQ Orbitrap Velos mass spectrometer (Thermo Fisher Scientific, Bremen, Germany). Ions between 190 and $600 \mathrm{~m} / \mathrm{z}$ were scanned in the orbitrap detector with a resolution of 30000 (maximum fill time $400 \mathrm{~ms}$, AGC target 106, lock mass $445.12003 \mathrm{~m} / \mathrm{z}$ ). Data analysis was performed using the Xcalibur ${ }^{\mathrm{TM}}$ Software (Thermo Scientific). The isolation width for the extracted ion chromatograms was $<50 \mathrm{ppm}$.

\section{ESI-MS analysis of derivatized CorB}

To elucidate the binding of $\mathbf{1}$ to CorB, electrospray ionization mass spectrometry (ESI-MS) was performed on a Synapt G2 mass spectrometer (Waters). Experiments were conducted with CorB and CorB incubated with a $10 \times$ molar excess of 1 . Prior to 
measurements, buffers were replaced with Milli-Q $\mathrm{H}_{2} \mathrm{O}$ by cycles of concentration and adding Milli-Q $\mathrm{H}_{2} \mathrm{O}$. Measurement of the samples was conducted by direct infusion in ESI positive mode with a capillary voltage of $3 \mathrm{kV}$, sampling cone of 30 and extraction cone of 3.0. The instrument was operated at a resolution of 20000 , a scan range from 50 to $2000 \mathrm{Da}$ and a scan time of $0.5 \mathrm{~s}$. Spectra of each infusion were combined.

\section{Protein crystallization}

Commercial screens were used to screen for crystallization conditions. All screens were set up at $20{ }^{\circ} \mathrm{C}$ and $4{ }^{\circ} \mathrm{C}$ in 96 well plates using the sitting drop vapor diffusion method. Based on initial hits fine screens were prepared at $20{ }^{\circ} \mathrm{C}$ using the hanging drop vapor diffusion method $(1 \mu \mathrm{L} \times 1 \mu \mathrm{L}$ drop size and $490 \mu \mathrm{L}$ reservoir volume). Best crystals grew overnight in crystallization buffer (0.1 M sodium cacodylate (pH 6.5), 8\% (w/ v) PEG8000 and 34\% (v/v) MPD). These crystals were harvested after 12-16 $\mathrm{h}$ and used for the structure determination of CorBred. To obtain the structure of CorB-1, we incubated the identical protein batch overnight with 1 and performed crystallization immediately.

\section{Structure determination and refinement}

Data sets were collected at the in-house X-ray source at $100 \mathrm{~K}$ using $0.5^{\circ}$ rotation and $300 \mathrm{~s}$ exposure time per image. All crystals were grown in conditions that included precipitant concentrations in the cryoprotectant range $(+30 \%$ (v/v) MPD. Data were reduced using the X-ray Detector Software (XDS) program package. ${ }^{\mathbf{4 8 , 4 9}}$ A sequence analysis using HHPRED ${ }^{50}$ revealed the homology of CorB to several proteins of the thiolase superfamily to which CorB shows a sequence identity of $21 \%$ to $33 \%$. Initial phases were obtained by molecular replacement procedure using PHASER $^{51}$ and a manually adjusted model of $\mathrm{PqsD}^{46}$ (pdb-code: $3 \mathrm{H78}$ ). Several cycles of reciprocal space rigid body refinement using REFMAC5 ${ }^{52}$ and real space rigid body refinement using COOT $^{53}$ improved the phase information and established an initial model of CorB. This model was improved through iterative cycles of refinement including simulated annealing and TLS parameterization ${ }^{54}$ using PHENIX ${ }^{55}$ and REFMAC5, and rebuilding of the structure with COOT. Geometric parameters were validated using Molprobity. ${ }^{56}$ Figures were generated and rendered using PYMOL. ${ }^{57}$

\section{Phylogenetic analysis}

A set of homologous ketosynthases was submitted to phylogenetic reconstruction. This set comprised the amino acid sequences of CorB, the homologous MyxB, and previously studied ketosynthases, ${ }^{17}$ as well as close homologs (Bit-Score $\geq$ 800) obtained from sequence similarity searches using BLAT ${ }^{58}$ (v34) in prokaryotic genomes available from NCBI. On this set of homologs we used MAFFT $^{59}$ (v6.851b) to generate a multiple sequence alignment (MSA) based on the chemical properties of the amino acid residues. Subsequently both a Maximum Likelihood (ML), with FastTree ${ }^{20}$ (v2.1.7), as well as a Maximum Parsimony (P), with phangorn ${ }^{21}$ (v1.99.11), phylogenetic trees were computed. The latter parsimonious approach obtained local confidence values using 100 bootstrapped versions of the MSA. Subsequently, using ape ${ }^{60}$ (v3.2), from both phylogenies a strict consensus tree was inferred. Based on this consensus tree, in a final step, we computed a tree with branch lengths applying the accelerated transformation (ACCTRAN) algorithm.

\section{Conclusions}

In conclusion, we found that the interconnecting PKS CorB possesses a typical thiolase fold, and we showed by site-directed mutagenesis that all members of the catalytic triad (C121, H264, N292) are required for catalysis. The catalytic triad residues of CorB are more distant from each other compared with structurally related enzymes that facilitate a decarboxylation reaction, and this separation is linked to a shift in helix $\alpha 5$ that is similar to that seen in HMG-CoA synthases. Interestingly, both CorB and HMG-CoA synthases have a wider active site compared with FabH and PKS. In CorB the active site is accessible from two directions, giving rise to a wide, T-shaped cavity. We could show that a substrate mimic occupies one side of the channel and we suggest that the remarkable wide T-shaped binding site of CorB is the key feature to accommodate two polyketide chains during catalysis to interconnect the substrates by a head-to-head Claisen-like condensation reaction.

The ability of CorB to catalyze chain fusions makes it a promising tool for semi-synthetic approaches, and opens up the possibility to use the enzyme as a tool in synthetic biology approaches. Future experiments will reveal the complete substrate flexibility of this pyrone-forming enzyme. ${ }^{61}$

\section{Acknowledgements}

Financial support came from the German Centre for Infection Research (DZIF), and from the German Research Foundation (Collaborative Research Center 766 and Research Unit FOR854). We gratefully acknowledge J. H. Sahner and R. Müller for supporting us with substrate 2. We thank students C. Kielkopf, A.-S. Ramms, D. Stehle, B. Marx, J. B. Heim, and M. Oelker for their contributions to protein purification and their crystallization trials, and M. Sylvester for MS measurements.

\section{Notes and references}

$\S$ Structure factor amplitudes and atomic coordinates were deposited in the Protein Data Bank with entry code 4YUF for CorB-ox and 4YUC for CorB-1 and 5C1J for CorB-red, respectively.

1 I. Chopra, L. Hesse and A. J. O'Neill, J. Appl. Microbiol., 2002, 92, 4S-15S.

2 C. Hertweck, Angew. Chem., Int. Ed. Engl., 2009, 48, 46884716.

3 J. W. Li and J. C. Vederas, Science, 2009, 325, 161-165.

4 O. Erol, T. F. Schaberle, A. Schmitz, S. Rachid, C. Gurgui, M. El Omari, F. Lohr, S. Kehraus, J. Piel, R. Muller and G. M. Konig, ChemBioChem, 2010, 11, 1253-1265. 
5 A. Schiefer, A. Schmitz, T. F. Schaberle, S. Specht, C. Lammer, K. L. Johnston, D. G. Vassylyev, G. M. Konig, A. Hoerauf and K. Pfarr, J. Infect. Dis., 2012, 206, 249-257.

6 J. Mukhopadhyay, K. Das, S. Ismail, D. Koppstein, M. Jang, B. Hudson, S. Sarafianos, S. Tuske, J. Patel, R. Jansen, H. Irschik, E. Arnold and R. H. Ebright, Cell, 2008, 135, 295-307.

7 K. Mariner, M. McPhillie, R. Trowbridge, C. Smith, A. J. O'Neill, C. W. Fishwick and I. Chopra, Antimicrob. Agents Chemother., 2011, 55, 2413-2416.

8 H. Irschik, R. Jansen, G. Hofle, K. Gerth and H. Reichenbach, J. Antibiot., 1985, 38, 145-152.

9 T. F. Schaberle, A. Schiefer, A. Schmitz, G. M. Konig, A. Hoerauf and K. Pfarr, Int. J. Med. Microbiol., 2014, 304, 72-78.

10 S. Brand, D. Holscher, A. Schierhorn, A. Svatos, J. Schroder and B. Schneider, Planta, 2006, 224, 413-428.

11 Y. Katsuyama, M. Matsuzawa, N. Funa and S. Horinouchi, J. Biol. Chem., 2007, 282, 37702-37709.

12 B. Shen, Curr. Opin. Chem. Biol., 2003, 7, 285-295.

13 S. J. Wakil, J. K. Stoops and V. C. Joshi, Annu. Rev. Biochem., 1983, 52, 537-579.

14 B. Middleton, Biochem. J., 1972, 126, 35-47.

15 A. M. Haapalainen, G. Merilainen and R. K. Wierenga, Trends Biochem. Sci., 2006, 31, 64-71.

16 C. Davies, R. J. Heath, S. W. White and C. O. Rock, Structure, 2000, 8, 185-195.

17 T. Bretschneider, G. Zocher, M. Unger, K. Scherlach, T. Stehle and C. Hertweck, Nat. Chem. Biol., 2012, 8, 154-161.

18 Q. L. He, X. Y. Jia, M. C. Tang, Z. H. Tian, G. L. Tang and W. Liu, ChemBioChem, 2009, 10, 813-819.

19 H. Sucipto, J. H. Sahner, E. Prusov, S. C. Wenzel, R. W. Hartmann, J. Koehnke and R. Muller, Chem. Sci., 2015, 6, 5076-5085.

20 M. N. Price, P. S. Dehal and A. P. Arkin, Mol. Biol. Evol., 2009, 26, 1641-1650.

21 K. P. Schliep, Bioinformatics, 2011, 27, 592-593.

22 S. W. Fuchs, K. A. Bozhuyuk, D. Kresovic, F. Grundmann, V. Dill, A. O. Brachmann, N. R. Waterfield and H. B. Bode, Angew. Chem., Int. Ed. Engl., 2013, 52, 4108-4112.

23 H. Sucipto, S. C. Wenzel and R. Muller, ChemBioChem, 2013, 14, 1581-1589.

24 L. Holm and C. Sander, Trends Biochem. Sci., 1995, 20, 478480.

25 K. S. Gajiwala, S. Margosiak, J. Lu, J. Cortez, Y. Su, Z. Nie and K. Appelt, FEBS Lett., 2009, 583, 2939-2946.

26 S. D. Pegan, K. Rukseree, G. C. Capodagli, E. A. Baker, O. Krasnykh, S. G. Franzblau and A. D. Mesecar, Biochemistry, 2013, 52, 912-925.

27 X. Qiu, A. E. Choudhry, C. A. Janson, M. Grooms, R. A. Daines, J. T. Lonsdale and S. S. Khandekar, Protein Sci., 2005, 14, 2087-2094.

28 X. Qiu, C. A. Janson, W. W. Smith, M. Head, J. Lonsdale and A. K. Konstantinidis, J. Mol. Biol., 2001, 307, 341-356.

29 M. B. Austin, M. E. Bowman, J. L. Ferrer, J. Schroder and J. P. Noel, Chem. Biol., 2004, 11, 1179-1194.
30 M. B. Austin, T. Saito, M. E. Bowman, S. Haydock, A. Kato, B. S. Moore, R. R. Kay and J. P. Noel, Nat. Chem. Biol., 2006, 2, 494-502.

31 K. Gokulan, S. E. O'Leary, W. K. Russell, D. H. Russell, M. Lalgondar, T. P. Begley, T. R. Ioerger and J. C. Sacchettini, J. Biol. Chem., 2013, 288, 16484-16494.

32 H. Morita, S. Kondo, S. Oguro, H. Noguchi, S. Sugio, I. Abe and T. Kohno, Chem. Biol., 2007, 14, 359-369.

33 S. B. Rubin-Pitel, H. Zhang, T. Vu, J. S. Brunzelle, H. Zhao and S. K. Nair, Chem. Biol., 2008, 15, 1079-1090.

34 M. He, M. Varoglu and D. H. Sherman, J. Bacteriol., 2000, 182, 2619-2623.

35 J. M. Jez, M. B. Austin, J. Ferrer, M. E. Bowman, J. Schroder and J. P. Noel, Chem. Biol., 2000, 7, 919-930.

36 J. G. Olsen, A. Kadziola, P. von Wettstein-Knowles, M. Siggaard-Andersen, Y. Lindquist and S. Larsen, FEBS Lett., 1999, 460, 46-52.

37 N. Campobasso, M. Patel, I. E. Wilding, H. Kallender, M. Rosenberg and M. N. Gwynn, J. Biol. Chem., 2004, 279, 44883-44888.

38 F. Pojer, J. L. Ferrer, S. B. Richard, D. A. Nagegowda, M. L. Chye, T. J. Bach and J. P. Noel, Proc. Natl. Acad. Sci. U. S. A., 2006, 103, 11491-11496.

39 N. Shafqat, A. Turnbull, J. Zschocke, U. Oppermann and W. W. Yue, J. Mol. Biol., 2010, 398, 497-506.

40 M. M. Alhamadsheh, F. Musayev, A. A. Komissarov, S. Sachdeva, H. T. Wright, N. Scarsdale, G. Florova and K. A. Reynolds, Chem. Biol., 2007, 14, 513-524.

41 F. Musayev, S. Sachdeva, J. N. Scarsdale, K. A. Reynolds and H. T. Wright, J. Mol. Biol., 2005, 346, 1313-1321.

42 M. B. Austin and J. P. Noel, Nat. Prod. Rep., 2003, 20, 79-110.

43 J. M. Jez, M. E. Bowman and J. P. Noel, Biochemistry, 2001, 40, 14829-14838.

44 A. T. Keatinge-Clay, D. A. Maltby, K. F. Medzihradszky, C. Khosla and R. M. Stroud, Nat. Struct. Mol. Biol., 2004, 11, 888-893.

45 C. N. Steussy, A. A. Vartia, J. W. Burgner 2nd, A. Sutherlin, V. W. Rodwell and C. V. Stauffacher, Biochemistry, 2005, 44, 14256-14267.

46 A. K. Bera, V. Atanasova, H. Robinson, E. Eisenstein, J. P. Coleman, E. C. Pesci and J. F. Parsons, Biochemistry, 2009, 48, 8644-8655.

47 A. Rentsch and M. Kalesse, Angew. Chem., Int. Ed. Engl., 2012, 51, 11381-11384.

48 W. Kabsch, Acta Crystallogr., Sect. D: Biol. Crystallogr., 2010, 66, 133-144.

49 W. Kabsch, Acta Crystallogr., Sect. D: Biol. Crystallogr., 2010, 66, 125-132.

50 M. Remmert, A. Biegert, A. Hauser and J. Soding, Nat. Methods, 2012, 9, 173-175.

51 A. J. McCoy, R. W. Grosse-Kunstleve, P. D. Adams, M. D. Winn, L. C. Storoni and R. J. Read, J. Appl. Crystallogr., 2007, 40, 658-674.

52 G. N. Murshudov, A. A. Vagin and E. J. Dodson, Acta Crystallogr., Sect. D: Biol. Crystallogr., 1997, 53, 240-255.

53 P. Emsley, B. Lohkamp, W. G. Scott and K. Cowtan, Acta Crystallogr., Sect. D: Biol. Crystallogr., 2010, 66, 486-501. 
54 M. D. Winn, M. N. Isupov and G. N. Murshudov, Acta Crystallogr., Sect. D: Biol. Crystallogr., 2001, 57, 122-133.

55 P. D. Adams, P. V. Afonine, G. Bunkoczi, V. B. Chen, I. W. Davis, N. Echols, J. J. Headd, L. W. Hung, G. J. Kapral, R. W. Grosse-Kunstleve, A. J. McCoy, N. W. Moriarty, R. Oeffner, R. J. Read, D. C. Richardson, J. S. Richardson, T. C. Terwilliger and P. H. Zwart, Acta Crystallogr., Sect. D: Biol. Crystallogr., 2010, 66, 213-221.

56 V. B. Chen, W. B. Arendall 3rd, J. J. Headd, D. A. Keedy, R. M. Immormino, G. J. Kapral, L. W. Murray,
J. S. Richardson and D. C. Richardson, Acta Crystallogr., Sect. D: Biol. Crystallogr., 2010, 66, 12-21.

57 Schrodinger, LLC, unpublished work.

58 W. J. Kent, Genome Res., 2002, 12, 656-664.

59 K. Katoh, K. Misawa, K. Kuma and T. Miyata, Nucleic Acids Res., 2002, 30, 3059-3066.

60 E. Paradis, J. Claude and K. Strimmer, Bioinformatics, 2004, 20, 289-290.

61 C. Hertweck, Trends Biochem. Sci., 2015, 40, 189-199. 